\title{
Laparoscopic colorectal surgery: why, when, how?
}

\author{
Gilles Manceau ${ }^{1} \cdot$ Yves Panis $^{1}$
}

Received: 23 December 2015/Accepted: 23 January 2016/Published online: 26 February 2016

(C) Italian Society of Surgery (SIC) 2016

Keywords Laparoscopy · Colorectal surgery · Cancer · Crohn's disease · Ulcerative colitis

\section{Introduction}

Since 1987 and the first laparoscopic cholecystectomy, laparoscopic surgery has spread tremendously in gastrointestinal surgery. Within a few years, there was widespread adoption of laparoscopy as the standard surgical approach in a growing number of pathologies. However, for colorectal surgery, the uptake of laparoscopy has been dramatically slower. One explanation is that laparoscopic colorectal resection is a technically complex procedure (colon and rectum mobilization, dissection in several abdominal quadrants, intracorporeal division of major vessels and ligation at their origins in case of malignancy, extraction of large specimens and performance of a bowel anastomosis), with a steep learning curve that requires a good surgical experience. However, the boundaries have been pushed back over the two last decades by growing experience of surgeons and technical advances, making laparoscopy the surgical approach of choice for colorectal resection in nearly all patients.

Yves Panis

yves.panis@aphp.fr

1 Department of Colorectal Surgery, Pôle des Maladies de l'Appareil Digestif (PMAD), Beaujon Hospital, Assistance Publique-Hôpitaux de Paris (AP-HP), University Denis Diderot (Paris VII), 100 Boulevard du Général Leclerc, 92110 Clichy, France
Why?

For colon cancer surgery, it is now well established by several phase III trials that laparoscopic resection is the recommended surgical approach. Compared to open surgery, laparoscopic resection has been demonstrated to improve short-term postoperative results with significant reduction in analgesic consumption, time to first flatus, time to oral intake tolerance and duration of hospital stay. In addition, laparoscopy reduces intraoperative blood loss, is associated with gains in cosmesis and faster recovery of physical function. More importantly, it has no detrimental effect on oncological outcomes. In 2008, a review from The Cochrane Collaboration with 12 randomized trials involving 3346 patients reported that the recurrence rates at the site of the primary tumor were equivalent between patients receiving open and laparoscopic surgery [1]. The occurrence of port-site/wound metastases was also similar. No difference was observed in terms of cancer-related and overall mortalities. Besides, the advantages of laparoscopic approach seem to be even more important in elderly patients, which represent the vast majority of patients treated for this type of cancer. Indeed, published studies that reported short-term outcomes after laparoscopic or open colorectal resection in the specific group of elderly patients suggest that the laparoscopic technique could be safely used in elderly patients with colon cancer. For this population, laparoscopy is associated with less postoperative morbidity compared with laparotomy, mostly by decreasing medical and cardiopulmonary complications [2]. Finally, laparoscopy could also have a positive impact on postoperative mortality. In a French survey of more than 84,000 patients who underwent colorectal resection for cancer, we reported that laparoscopic surgery was independently associated with a significant decrease in 
postoperative mortality in multivariate analysis $(\mathrm{OR}=0.59 ; 95 \%$ CI 0.54-0.65; $p<0.001)$ [3].

For rectal cancer, which presents a host of other technical challenges for laparoscopic approach (dissection in a narrow and deep space, oncological imperatives with total mesorectal excision, risk of pelvic nerve injury, etc.), evidence of the effectiveness of laparoscopic approach took longer to be proved. But recent high-quality studies and meta-analyses tend to reach the same conclusions with respect to the use of laparoscopy for this cancer type. Notably, long-term results of the noninferiority COLOR II trial showed that locoregional recurrence rate and diseasefree and overall survival were similar between laparoscopic and open surgery [4].

For inflammatory bowel disease, we have recently reported in a systematic review that laparoscopic ileal pouch-anal procedures for ulcerative colitis are associated with improved short-term outcomes (faster recovery of bowel function and shorter length of hospital stay), as well as a reduced rate of long-term small-bowel obstruction and hernia, compared with an open approach [5]. Furthermore, in case of ileal pouch-anal anastomosis for women of childbearing age with a desire for pregnancy, laparoscopy must be the preferred surgical approach [6]. Indeed, as this technique is associated with a reduction in adhesion formation, it decreases the risk of postoperative tubular infertility [7].

\section{When?}

For colon cancers without evidence of locoregional invasion, laparoscopy can be used in all elective cases. Although all randomized trials excluded adenocarcinomas of the transverse colon and the splenic flexure, it seems that laparoscopic resection is a safe and effective technique for these tumor locations. Several surgical teams, including ours, reported feasibility of laparoscopic resection in selected patients with $\mathrm{T} 4$ colon cancers requiring en bloc resection. However, for tumors with suspicion of adjacent organ invasion on preoperative CT scan, we believe that this surgical approach should be reserved in very expert hands. But in case of locally advanced tumors with abdominal wall invasion, laparoscopy has no detrimental effect on surgical and oncological outcomes. History of open abdominal surgery per se should not be a contraindication for laparoscopic approach. The fear of intense intra-abdominal adhesions is most often overestimated and the procedure can be achieved at the cost of a minor adhesiolysis. Nevertheless, patients with a large incisional hernia require an open approach, because of the need for hernia repair.
Laparoscopy should be the surgical approach of choice for Crohn's disease and ulcerative colitis in case of noncomplicated severe acute colitis for patients who do not respond to medical therapy [6]. It is associated with improved short-term outcomes in comparison with open surgery, in terms of postoperative infectious complications, postoperative recovery and length of hospital stay [5].

\section{How?}

In the field of minimally invasive surgery, the term "laparoscopic approach" includes different techniques. Colorectal resection by laparoscopy should be performed with multiple laparoscopic ports. Indeed, hand-assisted laparoscopic surgery with insertion of the surgeon's hand inside the abdominal cavity requires a larger incision and is associated with worse postoperative outcomes in comparison with multiple-port laparoscopic approach. Single-incision laparoscopic surgery (SILS) seems to be a good evolution of the conventional laparoscopic approach. With the use of a single multichannel port site, it provides the potential for minimizing surgical trauma. Several retrospective studies have emphasized that SILS is safe and feasible for a large number of indications in colorectal surgery. To date, only one randomized controlled trial has compared SILS and conventional laparoscopy in colon cancer surgery [8]. It found two potential advantages with the SILS technique, including decreased length of hospital stay and reduced postoperative pain. However, this study was of small sample size, with only 50 included patients, and did not provide any long-term oncological outcomes. Furthermore, SILS is associated with technical challenges, including dissection and exposure difficulties, even in the hands of surgeons with good experience in laparoscopic surgery. Finally, robotic-assisted laparoscopy is also an emerging and promising technique in colorectal surgery. But the ROLARR (RObotic Versus LAparoscopic Resection for Rectal Cancer) study, whose results were recently presented during the last ESCP congress, failed to demonstrate any benefit of this technique over the conventional laparoscopic approach in terms of conversion rate. Thus, although attractive, current evidence from the literature does not justify the significant costs of this technique.

Laparoscopic approach should be considered now as the standard of care in almost all colorectal diseases. In other words, colorectal surgeons should shift the paradigm and try to identify indications in which open surgery is necessary, rather than trying to identify good candidates for laparoscopy. 


\section{Compliance with ethical standards}

Conflict of interest The authors declare that they have no conflict of interest.

Ethical standard All procedures in our paper were in accordance with the ethical standards of the institutional as well as national research committee and with the 1964 Helsinki declaration and its later amendments.

Research involving human participants and/or animals This articles does not contain any studies with human participants or animal performed by any of the authors.

Informed consent For this type of study formal consent is not required.

\section{References}

1. Kuhry E, Schwenk WF, Gaupset R, Romild U, Bonjer HJ (2008) Long-term results of laparoscopic colorectal cancer resection. Cochrane Database Syst Rev 16(2):CD003432. doi:10.1002/ 14651858.CD003432.pub2

2. Allardyce RA, Bagshaw PF, Frampton CM, Frizelle FA, Hewett PJ, Rieger NA, Smith JS, Solomon MJ, Stevenson AR, Australasian Laparoscopic Colon Cancer Study Group (2010) Australasian Laparoscopic Colon Cancer Study shows that elderly patients may benefit from lower postoperative complication rates following laparoscopic versus open resection. $\mathrm{Br}$ J Surg 97(1):86-91. doi:10.1002/bjs.6785
3. Panis Y, Maggiori L, Caranhac G, Bretagnol F, Vicaut E (2011) Mortality after colorectal cancer surgery: a French survey of more than 84,000 patients. Ann Surg 254(5):738-743. doi:10.1097/SLA. 0b013e31823604ac (discussion 734-743)

4. Bonjer HJ, Deijen CL, Abis GA, Cuesta MA, van der Pas MH, de Lange-de Klerk ES, Lacy AM, Bemelman WA, Andersson J, Angenete E, Rosenberg J, Fuerst A, Haglind E, Group CIS (2015) A randomized trial of laparoscopic versus open surgery for rectal cancer. N Engl J Med 372(14):1324-1332. doi:10.1056/ NEJMoa1414882

5. Maggiori L, Panis Y (2013) Surgical management of IBD—from an open to a laparoscopic approach. Nat Rev Gastroenterol Hepatol 10(5):297-306. doi:10.1038/nrgastro.2013.30

6. Oresland T, Bemelman WA, Sampietro GM, Spinelli A, Windsor A, Ferrante M, Marteau P, Zmora O, Kotze PG, Espin-Basany E, Tiret E, Sica G, Panis Y, Faerden AE, Biancone L, Angriman I, Serclova Z, de Buck van Overstraeten A, Gionchetti P, Stassen L, Warusavitarne J, Adamina M, Dignass A, Eliakim R, Magro F, D'Hoore A, European Crohn's and Colitis Organisation (ECCO) (2015) European evidence based consensus on surgery for ulcerative colitis. J Crohns Colitis 9(1):4-25. doi:10.1016/j. crohns.2014.08.012

7. Beyer-Berjot L, Maggiori L, Birnbaum D, Lefevre JH, Berdah S, Panis Y (2013) A total laparoscopic approach reduces the infertility rate after ileal pouch-anal anastomosis: a 2-center study. Ann Surg 258(2):275-282. doi:10.1097/SLA.0b013e3182813741

8. Poon JT, Cheung CW, Fan JK, Lo OS, Law WL (2012) Singleincision versus conventional laparoscopic colectomy for colonic neoplasm: a randomized, controlled trial. Surg Endosc 26(10):2729-2734. doi:10.1007/s00464-012-2262-z 